SOLAR MOTION AND GALACTIC ROTATION:

RESULTS FROM THE LICK NORTHERN PROPER MOTION PROGRAM

\author{
Robert B. Hanson \\ Lick Observatory, University of California \\ Santa Cruz, California 95064, U.S.A.
}

\begin{abstract}
This paper reports the first results of solar motion and galactic rotation studies which are being done at Lick Observatory, using the new data from the Lick Northern Proper Motion (NPM) Program. This study finds:

(1) $\mathrm{A}=+11.31 \pm 1.06$ and $\mathrm{B}=-13.91 \pm 0.92 \mathrm{~km} / \mathrm{s} / \mathrm{kpc}$, consistent with a nearly flat galactic rotation curve with a local circular velocity near $200 \mathrm{~km} / \mathrm{s}$; (2) solar apex locations lying near the standard apex for low galactic latitudes, but trending strongly toward the direction of galactic rotation for high galactic latitudes; (3) RMS external error estimates for the Lick proper motions: 0 !'2/cent for the absolute zero-point in a typical NPM field, and $0 !^{\prime \prime} 06 \pm 0 .{ }^{\prime} 02 /$ cent for the overall systematic zero-point error. A more detailed presentation of these results has been given by Hanson (1987). The NPM program is reviewed in another paper in this Symposium, and has been described in detail by Klemola, Jones, and Hanson (1987).
\end{abstract}

\title{
1. DATA
}

The NPM astrometric reductions for absolute proper motions and positions have been completed for 617 NPM fields, almost completely covering the sky more than $\sim 10^{\circ}$ from the galactic plane between declinations $-3^{\circ}$ and $+68^{\circ}$. To test the accuracy of the NPM proper motions, and to begin their use in galactic structure studies, we have done solutions for galactic rotation and solar motion. The data used were the mean absolute proper motions of the 16th-magnitude "faint anonymous stars" in each field; these comprise in total about 60,000 stars, referred to about 40,000 galaxies. These stars are a well-defined statistical sample, selected at random within a limited magnitude range $(15<B<17)$, and uniformly distributed on the sky. The RMS precision of the NPM proper motion of an individual faint star is $\sim 0.5 /$ cent, for an epoch difference approaching 30 years (Klemola, Jones, and Hanson 1987).

\section{METHOD}

Klemola and Vasilevskis (1971) used the stellar absolute proper motions determined in the Lick Pilot Program (Klemola, Vasilevskis, Shane, and Wirtanen 1971) to solve for solar motion and galactic rotation, using the standard method 
developed by Vyssotsky and Williams (1948). This method uses the observed proper motions in right ascension and declination to solve for five unknowns: the three rectangular components of the solar motion, and the two Oort constants. Klemola and Vasilevskis (1971) made a number of solutions, some over the whole sky, and others dividing the sky into galactic latitude zones. For this study we have done extensive test solutions using the new NPM data to devise an improved version of this method, which: (1) solves simultaneously for the galactic rectangular components $(\mathrm{X}, \mathrm{Y}, \mathrm{Z})$ of the solar motion in several galactic latitude zones, and for the overall values of the Oort rotation constants (A,B); and (2) tests the overall zero-point calibration of the NPM absolute proper motions. Details of the solution method, and full results of these test solutions are given by Hanson (1987).

\section{TEST SOLUTIONS}

The first major result of these tests was that small zero-point terms (on the order of $0.3 \mu \mathrm{m}$, or 0 !'06/cent) needed to be included in the model. Tests without the zero-point terms gave substantially larger unit weight errors; furthermore the correlations between terms were such that biased values for the Oort constants were obtained when the zero-point terms were not properly taken into account. Illustrating this, Table I shows results of the two best solutions with and without zero-point terms $\left(\mathrm{K}_{\mathrm{x}}, \mathrm{K}_{\mathbf{y}}\right)$. Each solution used six galactic latitude bins. The solution without zero-point terms is the preliminary solution reported in van Altena et al. (1986). The solution with zero-point terms is the "adopted" solution, with 6 galactic latitude zones, as will be discussed below. We note in particular that the values of Oort's B from the two solutions differ by three times their mean error. These effects are caused by correlations (due to incomplete sky coverage) between the zero-point terms and the galactic rotation terms.

Table I. Galactic rotation solutions with and without zero-point terms.

\begin{tabular}{|c|c|c|c|}
\hline & $\begin{array}{l}\text { Unit Weight } \\
\text { Error }(\mu \mathrm{m})\end{array}$ & $\mathrm{K}_{\mathbf{x}}{ }_{(\mu \mathrm{m})}^{\mathrm{K}_{\mathbf{y}}}$ & $\underset{(\mathrm{km} / \mathrm{s} / \mathrm{kpc})}{\mathrm{A})}$ \\
\hline $\begin{array}{l}\text { without } \\
\left(\mathrm{K}_{\mathbf{x}}, \mathrm{K}_{\mathbf{y}}\right)\end{array}$ & 0.987 & & $\begin{array}{r}+10.98-11.16 \\
\pm 0.89 \pm 0.80\end{array}$ \\
\hline $\begin{array}{l}\text { with } \\
\left(\mathrm{K}_{\mathbf{x}}, \mathrm{K}_{\mathrm{y}}\right)\end{array}$ & 0.971 & $\begin{array}{ll}+0.322 & -0.108 \\
\pm 0.052 & \pm 0.219\end{array}$ & $\begin{array}{l}+11.31-13.91 \\
\pm 1.06 \pm 0.92\end{array}$ \\
\hline
\end{tabular}

The second major result of the test solutions was that the proper motion data must be binned by at least 5 or 6 galactic latitude zones in order to solve for the solar motion. This is due to the substantial variation in the solar motion with distance from the galactic plane, and hence with galactic latitude ( $c f$. van Altena 1974). Models with too few galactic latitude bins showed strong bias in the Oort constants (A,B), and deceptively small standard errors. 
We thus adopt the solution with zero-point terms and 6 galactic latitude zones (Table I, line 2) as giving the most reliable results for galactic rotation and solar motion from the NPM data. The unit weight error in this solution yields an external error estimate for the RMS error $\sigma_{\circ}$ of the absolute zero-point in a typical NPM field; this corresponds to $0 !^{\prime} 20 /$ cent over the average epoch span (27 years) of the NPM program, in excellent agreement with results previously found from NPM overlap tests (Klemola, Jones, and Hanson 1987).

\section{GALACTIC ROTATION}

The galactic rotation results obtained here are preliminary in the sense that only a small subset of the total NPM proper motion data has so far been used. Nonetheless, the present results are highly significant; indeed the new determination of the Oort galactic rotation constants yields some of the most precise results ever obtained from proper motions. This is due to the high precision of the NPM proper motions and the very large numbers of stars involved.

Kerr and Lynden-Bell (1986) have made a comprehensive review of recent determinations of the galactic constants. The value determined from the NPM data for the Oort constant $\mathrm{A}$ is

$$
\mathrm{A}=+11.31 \pm 1.06 \mathrm{~km} / \mathrm{s} / \mathrm{kpc}
$$

somewhat smaller than the mean value, $+14.4 \pm 1.2$, tabulated by Kerr and Lynden-Bell (1986, Table 10). Until more detailed studies can be made, we would not attatch any great significance to this difference. For the Oort constant B, the NPM result is

$$
\mathrm{B}=-13.91 \pm 0.92 \mathrm{~km} / \mathrm{s} / \mathrm{kpc}
$$

in good agreement with the mean value, $-12.0 \pm 2.8$, of Kerr and Lynden-Bell. Calculating the errors including the correlation $(-0.23)$ between the NPM values of $\mathrm{A}$ and $\mathrm{B}$, we obtain for the local angular velocity

$$
\omega_{\circ}=(A-B)=+25.2 \pm 1.6 \mathrm{~km} / \mathrm{s} / \mathrm{kpc} \text {. }
$$

Adopting Kerr and Lynden-Bell's value $R_{\circ}=8.5 \pm 1.1 \mathrm{kpc}$ for the distance to the galactic center, the NPM results imply a local circular velocity

$$
\Theta_{\circ}=R_{\circ}(A-B)=214 \pm 31 \mathrm{~km} / \mathrm{s}
$$

in good agreement with recent determinations (Kerr and Lynden-Bell 1986, Table 4). For the local value for the slope of the galactic rotation curve, the NPM results give

$$
(\mathrm{d} \Theta / \mathrm{dR})_{\mathrm{o}}=-(\mathrm{A}+\mathrm{B})=+2.6 \pm 1.2 \mathrm{~km} / \mathrm{s} / \mathrm{kpc} .
$$

Although the positive value of $-(\mathrm{A}+\mathrm{B})$ formally implies a rising local rotation curve, at this stage of the NPM analysis we would not consider the two-sigma difference from a zero value (flat rotation curve, $c f$. Kerr and Lynden-Bell, Sec. 8.2) to be highly significant. 
Table II. Adopted solution: solar motion

(galactic rectangular coordinates," /cent) vs. galactic latitude.

\begin{tabular}{|c|c|c|c|c|c|c|c|}
\hline Zone & $|b|$ & $\langle|\mathrm{b}|\rangle$ & Fields & $\mathrm{X}$ & $\mathrm{Y}$ & Z & Total \\
\hline 1 & $0^{\circ}-20^{\circ}$ & $15.9^{\circ}$ & 69 & $\begin{array}{l}+0.174 \\
\pm 0.044\end{array}$ & $\begin{array}{l}+0.374 \\
\pm 0.055\end{array}$ & $\begin{array}{l}+0.136 \\
\pm 0.040\end{array}$ & $\begin{array}{r}0.435 \\
\pm 0.050\end{array}$ \\
\hline 2 & $20^{\circ}-30^{\circ}$ & $24.8^{\circ}$ & 115 & $\begin{array}{l}+0.163 \\
\pm 0.038\end{array}$ & $\begin{array}{l}+0.530 \\
\pm 0.049\end{array}$ & $\begin{array}{l}+0.162 \\
\pm 0.034\end{array}$ & $\begin{array}{r}0.577 \\
\pm 0.047\end{array}$ \\
\hline 3 & $30^{\circ}-40^{\circ}$ & $34.8^{\circ}$ & 112 & $\begin{array}{l}+0.241 \\
\pm 0.036\end{array}$ & $\begin{array}{l}+0.614 \\
\pm 0.049\end{array}$ & $\begin{array}{l}+0.165 \\
\pm 0.033\end{array}$ & $\begin{array}{r}0.680 \\
\pm 0.044\end{array}$ \\
\hline 4 & $40^{\circ}-50^{\circ}$ & $44.9^{\circ}$ & 100 & $\begin{array}{l}+0.219 \\
\pm 0.035\end{array}$ & $\begin{array}{l}+0.736 \\
\pm 0.048\end{array}$ & $\begin{array}{l}+0.183 \\
\pm 0.034\end{array}$ & $\begin{array}{r}0.790 \\
\pm 0.044\end{array}$ \\
\hline 5 & $50^{\circ}-60^{\circ}$ & $54.6^{\circ}$ & 95 & $\begin{array}{l}+0.209 \\
\pm 0.035\end{array}$ & $\begin{array}{l}+0.806 \\
\pm 0.049\end{array}$ & $\begin{array}{l}+0.150 \\
\pm 0.039\end{array}$ & $\begin{array}{r}0.846 \\
\pm 0.045\end{array}$ \\
\hline 6 & $60^{\circ}-90^{\circ}$ & $68.1^{\circ}$ & 126 & $\begin{array}{l}+0.219 \\
\pm 0.035\end{array}$ & $\begin{array}{l}+0.902 \\
\pm 0.048\end{array}$ & $\begin{array}{l}+0.190 \\
\pm 0.055\end{array}$ & $\begin{array}{r}0.947 \\
\pm 0.044\end{array}$ \\
\hline
\end{tabular}

\section{SOLAR MOTION}

Table II presents the solar motion results for the six-zone solution adopted after the tests described above. The galactic rectangular components $(\mathrm{X}, \mathrm{Y}, \mathrm{Z})$ of the solar motion, in "/cent, are aligned with the standard (U,V,W) velocity coordinates (toward the galactic center, toward galactic rotation, toward the North galactic pole, respectively). The NPM faint stars exhibit a strong increase in the size of the solar motion (secular parallax) with galactic latitude, from $0 . " 4 /$ cent at $\langle|\mathrm{b}|\rangle=16^{\circ}$, to 0.9 / cent at $\langle|\mathrm{b}|\rangle=68^{\circ}$. This is qualitatively as expected (cf. van Altena 1974, Table III ) due to the inverse relationship between mean distance and galactic latitude (at a given apparent magnitude). Contrary to expectation, however, virtually all of the increase in the secular parallax of the NPM faint stars is due to the $\mathrm{Y}$ component, with little or no increase in the $\mathrm{X}$ or $\mathrm{Z}$ components. This suggests that both the kinematics and the distances of the NPM faint stars may be quite different from the standard assumptions which went into van Altena's (1974) galactic model.

Figure 1 shows the solar apex for the six galactic latitude zones in the NPM study. For each zone, the apex for the NPM faint stars lies directly between the standard solar apex and the direction of galactic rotation, as is the case for most groups of stars (cf. Mihalas and Binney 1981, Table 6-3). The Lick Pilot Program apex, however, lies some $10^{\circ}$ North of the new NPM values. The tendency of the Pilot Program results to fall farther North than expected (cf. Klemola and Vasilevskis 1971, Fig. 1) has been known since the first Lick results were reported by Vasilevskis and Klemola (1970). This effect may well be due to small zero- 
point errors in the Pilot Program proper motions.

The NPM results in Fig. 1 clearly show the large variation of the solar motion with galactic latitude. Even the lowest latitude zone $\left(\langle|b|\rangle=16^{\circ}\right)$ apex is $10^{\circ}$ from the standard apex; the variation with galactic latitude is nearly monotonic, with the highest latitude zone $\left(\langle|b|\rangle=68^{\circ}\right)$ apex displaced nearly two-thirds of the way toward the direction of galactic rotation. We interpret this result to mean that the 16th-magnitude stars in the NPM survey show a progressive lag behind circular galactic rotation; the lag increases with galactic latitude (i.e, with distance from the galactic plane), most likely due to the changing mixture of kinematic populations with increasing distance from the plane.

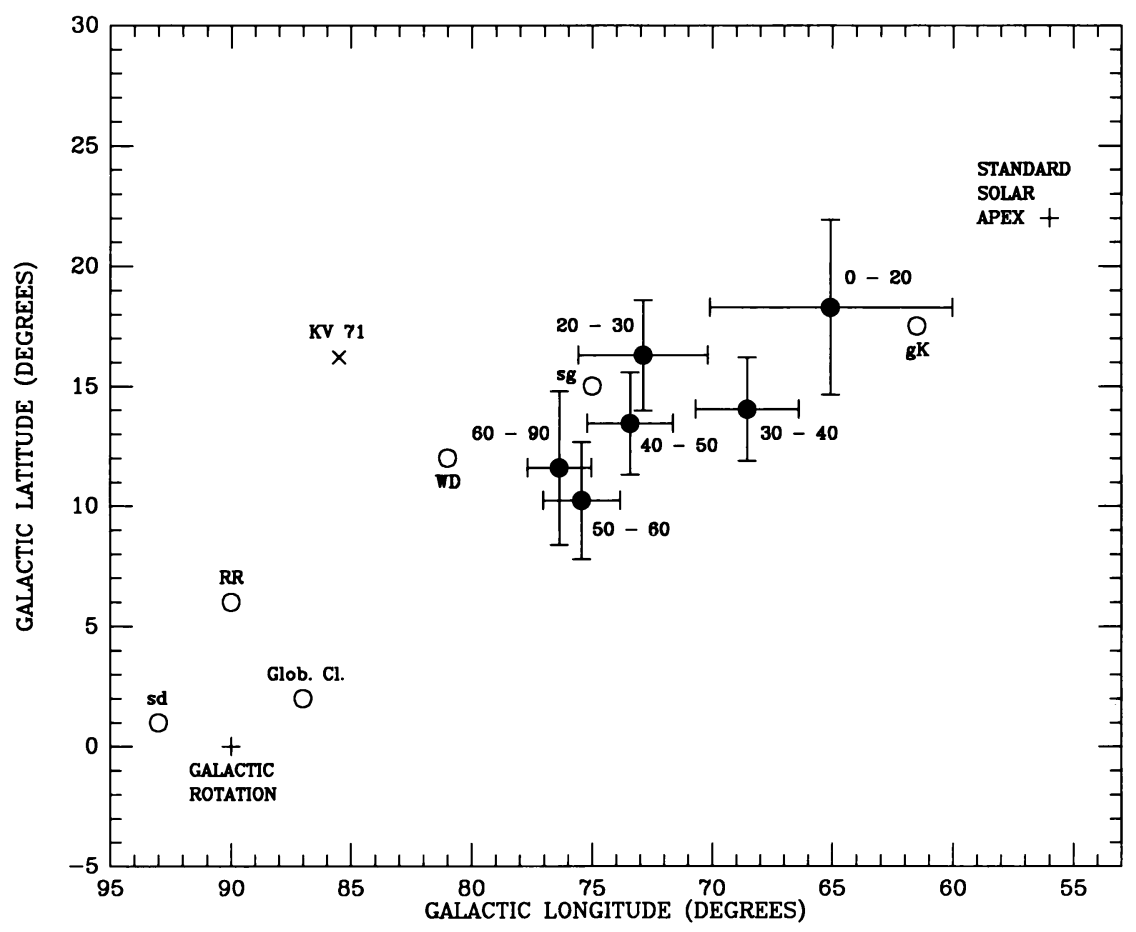

FIGURE. 1. Solar apex (in galactic coordinates) for the 16th-magnitude NPM stars, for the six galactic latitude zones adopted in the NPM study. For each latitude zone, the filled circles show the apex position, the error bars show the standard errors, and the ranges of $|\mathrm{b}|$ are given. The crosses mark the standard solar apex $(1, \mathrm{~b})=\left(56^{\circ},+22^{\circ}\right)$ and the direction of galactic rotation $(1, \mathrm{~b})=\left(90^{\circ}, 0^{\circ}\right)$. The open circles show the apex values (from Table 6-3 of Mihalas and Binney 1981) for several representative groups of stars: sd = subdwarfs, $R R=R R$ Lyrae variables with $\mathrm{P}>0.45$ days, Glob. cl. = globular clusters, $\mathrm{WD}=$ white dwarfs, $\mathrm{sg}=$ subgiants, $\mathrm{gK}=\mathrm{K}$ giants. The $\times$ symbol marked "KV 71" shows the solar apex determined by Klemola and Vasilevskis (1971, Table VII) for the 16thmagnitude stars in the Lick Pilot Program (Klemola et al. 1971). 
Qualitatively, this interpretation is consistent with the finding (Table II) that most of the increase in the secular parallax of the NPM faint stars is in the $\mathrm{Y}$ component (toward galactic rotation). The fact that the $\mathrm{X}$ and $\mathrm{Z}$ components show so little increase with galactic latitude indicates that the NPM faint stars' distances must scarcely decrease at all with increasing latitude. Thus the 16thmagnitude stars at high latitudes may in fact be more distant (and thus farther from the plane) than van Altena's (1974) model suggests.

This study points the way to further applications of the Lick NPM data, including studies of the stellar density and solar motion as they vary with distance from the galactic plane. Future work will include data from the declination zones from $+70^{\circ}$ to $+90^{\circ}$ and from $-5^{\circ}$ to $-20^{\circ}$ as they become available as the NPM program nears completion.

\section{ACKNOWLEDGEMENTS}

The Lick NPM program is supported by grants from the U.S. National Science Foundation. The results reported here include much work by A. R. Klemola and B. F. Jones.

\section{REFERENCES}

Hanson, R. B. (1987). Astron. J. 93, in press.

Kerr, F. J., and Lynden-Bell, D. (1986) Mon. Not. R. Astr. Soc. 221, 1023.

Klemola, A. R., Jones, B. F., and Hanson, R. B. (1987). Astron. J. 93, in press.

Klemola, A. R., and Vasilevskis, S. (1971). Publ. Lick Obs. 22, Part 3.

Klemola, A. R., Vasilevskis, S., Shane, C. D., and Wirtanen, C. A. (1971). Publ. Lick Obs. 22, Part 2.

Mihalas, D., and Binney, J. (1981). Galactic Astronomy, 2nd Edition.

(W. H. Freeman, San Francisco).

van Altena, W. F. (1974). Astron. J. 79, 826.

van Altena, W. F., Girard, T., López, C. E., Klemola, A. R., Jones, B. F., and Hanson, R. B. (1986). Highlights of Astronomy 6, 89.

Vasilevskis, S., and Klemola, A. R. (1970). In Proper Motions, I.A.U. Colloq. No. 7, edited by W. J. Luyten (University of Minnesota, Minneapolis), $\mathrm{p}$. 167.

Vyssotsky, A. N., and Williams, E. T. R. (1948). Publ. McCormick Obs. 10. 